\title{
Can psychedelics have a role in psychiatry
}

\section{once again?}

\author{
BEN SESSA
}

Psychedelic or hallucinogenic drugs such as lysergic acid diethylamide (LSD), 3,4,5trimethoxy- $\beta$-phenethylamine (mescaline), psilocybin, 3,4-methylenedioxymethamphetamine (MDMA), N,N-dimethyltryptamine (DMT) and their relations occur in abundance throughout the natural world, and have been used by humankind for thousands of years.

In some cultures they are important tools for spiritual experiences, whereas in others they are labelled as dangerous drugs of misuse. What is less well known about these substances is the role they played in psychiatry for a brief historical interval. This article offers a short overview of this period and questions whether interest in these compounds might be emerging again.

\section{CURRENT TRAINING AND KNOWLEDGE ABOUT PSYCHEDELICS}

Despite their history, psychedelics have dropped out of psychiatric dialogue for today's trainee psychiatrists (Strassman, 2001). In my own training, references to compounds like LSD, psilocybin and MDMA were usually followed by statements such as 'have no medical use'. But I was taught about the acute emergencies and social problems associated with their misuse.

Yet in the years between the first synthesis of LSD in the 1930s and the disappearance of psychedelic research by the late 1960 s, there was a furious growth of scientific interest in these substances. Many pioneers gave their careers to this field, hoping that psychedelic drugs could be to psychiatry what the microscope is to biology or the telescope is to astronomy: an essential tool to explore the parts of the internal world that are usually inaccessible (Grof, 2001).

\section{HISTORY OF PSYCHEDELIC RESEARCH}

The Swiss chemist, Albert Hoffman first synthesized LSD-25 while studying derivatives of the fungus ergot for use as potential medicines. When he accidentally absorbed some LSD during a laboratory session there followed an intense experience of perceptual and emotional effects (Hoffman, 1980).

By the late 1940s psychiatrists were beginning to experiment with LSD as a tool, and in 1951 it was the subject of a presentation at the annual conference of the American Psychological Association. Initial work explored the possibility that psychedelics might be used as 'psychotomimetics', to mimic the mental states of patients with schizophrenia (Osmond, 1957), and many health professionals were encouraged to partake in self-discovery or shared psychedelic experiences with their patients. Other research looked into using psychedelic drugs as adjuncts to psychotherapy. The therapy took the form of two broad types: first, psycholytic ('mind loosening') psychotherapy involved taking low doses of LSD as part of ongoing psychoanalytical therapy. The drug had a loosening effect and facilitated the exploration of repressed material. The second type, psychedelic ('mind manifesting') psychotherapy involved preparation sessions without LSD, then one single large-dose session that encouraged an intense reaction, followed by further non-drug sessions to explore the meaning of the material that emerged (Grinspoon \& Bakalar, 1997).

By 1965 over 2000 papers had been published describing positive results for over 40000 patients who took psychedelic drugs with few side-effects and a high level of safety (Masters \& Houston, 1970). The techniques were applied to the treatment of anxiety disorders, obsessivecompulsive disorders, depression, bereavement reactions and sexual dysfunction, among others (Newland, 1962; Grof,
2001). In the treatment of addiction, repeated controlled experiments demonstrated a consistent recovery and 6-month abstinence from drinking in $50-90 \%$ of participants after brief psychedelic therapy (Abramson, 1967; Hoffer, 1970). Another area where therapy was used successfully was in relieving pain and anxiety in terminal cancer (Kast, 1964).

\section{PROBLEMS WITH PREVIOUS} RESEARCH

Despite the volume of publications from this period, most of the published material refers to anecdotal case reports that are of little value by contemporary research standards because they lack sufficient follow-up and control participants (Grob, 1994). Even though results appeared promising, by the 1970 s, under pressure from the US justice department, virtually all research had ended. LSD had leaked from the scientific community to a wider audience. By 1966 LSD misuse had become a problem and its possession was made illegal. This prompted the scientific community to distance themselves from interest in such substances. Governments clamped down on research licences, and increasing reports of adverse reactions to psychedelics taken recreationally as opposed to those used in controlled, scientific circumstances (which remained safe) appeared in the literature (Strassman, 2001). As a result, research use ceased while illicit use remained, fuelled by a growing criminal distribution and financial system.

Until very recently, research on psychedelic drugs has been severely restricted, which explains the current lack of knowledge among psychiatrists.

\section{CURRENT RESEARCH}

Since the 1970s, MDMA psychotherapy has seen an emerging underground use by analysts. MDMA, strictly speaking an 'empathogen' rather than a psychedelic drug, is less intense and shorter-acting than LSD. It appears to offer a similar therapeutic potential for lowering a patient's defences and aiding the psychotherapeutic process (Holland, 2001).

A lifting of the government ban on psychedelic research in Switzerland between 1988 and 1993 allowed a brief recommencement of psycholytic psychotherapy using LSD and MDMA for patients with 
personality disorders, affective disorders and adjustment disorders. There are currently projects under development in Spain, Israel and the USA looking at MDMA-assisted psychotherapy in the treatment of post-traumatic stress disorder and as a treatment for anxiety and depression associated with cancer. Between 1990 and 1995 extensive studies of DMT, a strong but short-acting agent, were conducted with human participants in the USA (Strassman, 2001). Other research includes a double-blind placebo controlled study in Russia using ketamine in the treatment of heroin addiction, which has demonstrated improved rates of abstinence, maintained at 2-year follow-up (Krupitsky et al, 2002). Also in progress are studies looking at psilocybin in the treatment of obsessivecompulsive disorder and for reducing anxiety and pain in cancer patients. All of this research is well summarised on the Multidisciplinary Association for Psychedelic Studies (MAPS) website (www.maps.org).

\section{ONGOING PROBLEMS WITH CURRENT RESEARCH}

Although drug misuse remains a growing phenomenon in our global society the public and governments are suspicious of psychedelic research. The image of psychedelics, severely damaged by the 1960s drug culture, is further spoiled by drug use in today's 'rave' scene. Finding unbiased information about psychedelic research is often difficult.

However, many of the early pioneers of psychedelic research continue to promote it for the field of mental health. Dr Humphrey Osmond, the British psychiatrist who, in communication with the author Aldous Huxley, coined the term 'psychedelic' in the 1950s, strongly supported psychedelic research until his death last year aged 86 years, when he received a fitting tribute in the $B M J$ (Hopkins Tanne, 2004). Albert Hoffman, who

BEN SESSA, MBBS, BSc, MRCPsych, The Park Hospital, Old Road, Headington, Oxford OX3 7LQ, UK. E-mail: drbensessa@hotmail.com

(First received 27 January 2004, final revision 12 November 2004, accepted 21 November 2004)

celebrated his 99th birthday this year, maintains contact with organisations promoting scientific research into medical uses for psychedelic drugs, such as MAPS and the Heffter Research Institute (www.heffter.org).

Researchers believe these drugs are important tools for further academic study. Their recognised psychological effects fit well into an approach looking for the neurobiological links between mental and physical states. Also from a clinical point of view, the practice of traditional psychedelic psychotherapy - using the drugs as an adjunct to brief, time-limited psychotherapy - has much in common with the current practice of cognitive-behavioural therapy.

\section{CONCLUSION}

Perhaps it is surprising that there remains such considerable ignorance about the potential of these substances from within psychiatry itself. As with Galileo's telescope and Darwin's suggestion of our ascendancy from apes, radical scientific challenges tend to take the form of an attack on the anthropocentric model of the world. In the light of this, research that explores alternative states of consciousness and then offers a viable neurobiological substrate for the very human experience of religious encounter is bound to meet with objection from a generation of psychiatrists who have been conditioned to consider such work as 'mysticism'. Perhaps a more dispassionate criticism based upon scientific reasoning and not influenced by social or political pressures is called for if we are truly to investigate whether these substances can have a useful role in psychiatry today.

\section{DECLARATION OF INTEREST}

None.

\section{REFERENCES}

Abramson, H. A. (1967) The Use of LSD in Psychotherapy and Alcoholism. New York: Bobbs-Merrill.

Grinspoon, L. \& Bakalar, J. B. (1997) Psychedelic Drugs Reconsidered. New York: Lindesmith Center.

Grob, C. (1994) Psychiatric research with hallucinogens: what have we learned? In Yearbook for Ethnomedicine (eds C. Ratsch \& J. Baker). Berlin: Verlag für Wissenschaft und Bildung.

Grof, S. (200I) LSD Psychotherapy. Sarasota, FL: Multidisciplinary Association for Psychedelic Studies.

Hoffer, A. (1970) Treatment of alcoholism with psychedelic therapy. In Psychedelics, The Uses and Implications of Hallucinogenic Drugs (eds B. Aaronson \& H. Osmond). London: Hogarth Press.

Hoffman, A. (1980) LSD: My Problem Child. London: McGraw-Hill.

Holland, J. (200I) Ecstasy: The Complete Guide. Rochester, VT: Park Street Press.

Hopkins Tanne, J. (2004) Obituary for Humphrey Osmond. BM/, 328, 713

Kast, E. (1964) Pain and LSD-25: A theory of attenuation and anticipation. In LSD: The Consciousness Expanding Drug (ed. D. Solomon), pp. 24I-256. New York: GP Putman.

Krupitsky, E., Burakov, A. \& Romanova, T. (2002) Ketamine psychotherapy for heroin addiction. Journal of Substance Abuse Treatment, 23, 273-283.

Masters, R. E. L. \& Houston, J. (1970) Therapeutic applications of LSD and related drugs. In The Uses and Implications of Hallucinogenic Drugs (eds B. Aaronson \& H. Osmond). London: Hogarth Press.

Newland, C. (1962) My Self and I. New York: The New American Library.

Osmond, H. (1957) A review of the clinical effects of psychotomimetic agents. Annals of the New York Academy of Sciences, 66, 4I8-434.

Strassman, R. (200I) DMT: The Spirit Molecule. Rochester, VT: Park Street Press. 